\title{
Plus qu'un simple changement du nom allemand
}

\section{Julia Dratva}

Prof. Dr méd., présidente de la Société suisse de prévention et santé publique, ZHAW Département de la santé, institut des sciences de la santé

La Société suisse des médecins spécialistes en prévention et santé publique uniformise dès à présent son nom dans toutes les régions linguistiques. Public Health vient remplacer l'ancienne désignation de Gesundheitswesen en allemand. Mais derrière ce nouveau nom se cache plus qu'un simple changement de termes. Depuis 2015, la société a fait peau neuve. Il faut donc comprendre: new public health!

La Société suisse de prévention et santé publique est la faîtière des médecins spécialistes en prévention et santé publique, ainsi que de nombreux médecins actifs dans le domaine de la santé publique, qui adhèrent à une charte commune. Elle s'est constituée en association en 1976

$\mathrm{Au}$ cours de ces dernières années, initialement sous l'impulsion de sa présidente Dr méd. Karin Faisst, puis dès 2016 du Prof. Dr méd. Julia Dratva, la société a fait peau neuve et mis en œuvre d'importants développements et activités. Dans ce contexte, la Société a changé de nom et s'appelle à présent Société suisse de prévention et santé publique en français, en allemand, en italien et en anglais. Ce changement marque la volonté de notre organisation spécialisée de s'engager en faveur de la santé de l'ensemble de la population en Suisse, sur le plan sociétal, politique et de politique corporative.

\section{Au cours de ces dernières années, la société a fait peau neuve.}

Le système de santé est confronté à d'importants défis au vu des développements démographiques et de l'augmentation des maladies chroniques, du manque de personnel qualifié et de l'augmentation des coûts. Pour faire face à cette pression croissante sur le système de santé et sur la population ont été développées des stratégies de la santé en Suisse, telles que la stratégie NMT, le Plan d'action pour la prévention du suicide, la stratégie Santé2020 ou les nouveaux modèles de soins (p. ex. les soins intégrés). Ces stratégies adoptent une approche globale de la santé, en prenant en considération l'influence des facteurs physiques, psychiques, sociaux et environnementaux, ainsi que les différentes phases de la vie. Elles s'appuient sur les "triple aims" de Berwick et al. (2008), qui définissent trois objectifs supérieurs: 1. l'amélioration de la satisfaction du patient et de la qualité des soins (improving the experience of care, including quality and satisfaction), 2. l'amélioration de la santé des populations (improving the health of populations), et 3 . la réduction des coûts de la santé (reducing per capita costs of health care) (Berwick 2008).

Nous considérons que notre tâche centrale est d'assurer la formation continue des médecins.

Nous considérons que notre tâche centrale est d'assurer la formation continue des médecins dans le but de relever ces défis et de contribuer aux solutions dans le domaine de la santé publique. C'est la raison pour laquelle notre société spécialisée s'engage en faveur d'une formation continue innovante et adaptée aux besoins. En observant ce qu'il se passe dans d'autres pays européens, on constate rapidement l'efficacité accrue qui peut être atteinte grâce à des compétences en santé publique chez les médecins; en Suisse, nous devons encore travailler à la reconnaissance et la mise en œuvre d'une telle approche. L'innovation en matière de formation continue ne signifie pas seulement d'expérimenter de nouvelles approches, mais aussi, ou surtout, de donner aux médecins des outils pour relever les importants défis qui nous attendent. C'est dans cette optique que les experts, qui ont récemment procédé à l'accréditation de notre spécialité, ont demandé de renforcer encore davantage l'approche de «global public health» dans la formation continue.

Environ 40 pourcents des personnes ayant obtenu un titre en prévention et santé publique travaillent au 
sein des hautes écoles (universités et hautes écoles spécialisées), 40 autres pourcents sont actifs dans les institutions publiques telles que les services cantonaux ou scolaires de la santé, enfin 20 pourcents œuvrent au sein de ligues de la santé, de compagnies d'assurance ou d'autres institutions de santé publique. Au regard de cette diversité des champs d'activité, notre organisation se réjouit de la multiplication des sites extra-

\section{La formation continue exige une base d'au moins deux années d'activité clinique.}

universitaires de formation continue, tendance qui reflète mieux la pluralité des environnements dans lesquels travaillent les médecins spécialistes.

La formation continue spécifique à la branche des médecins spécialistes en prévention et santé publique se distingue par certaines particularités qui caractérisent ses titulaires par rapport à d'autres experts dans le domaine des soins. La formation continue exige une base d'au moins deux années d'activité clinique. Connaître la responsabilité du médecin envers les patients ainsi qu'avoir une vraie expérience du secteur des soins, constitue une force évidente des médecins en santé publique par rapport à d'autres spécialistes de la santé publique. La formation continue théorique spécifique à la branche implique, en règle générale, l'obtention du Master of Public

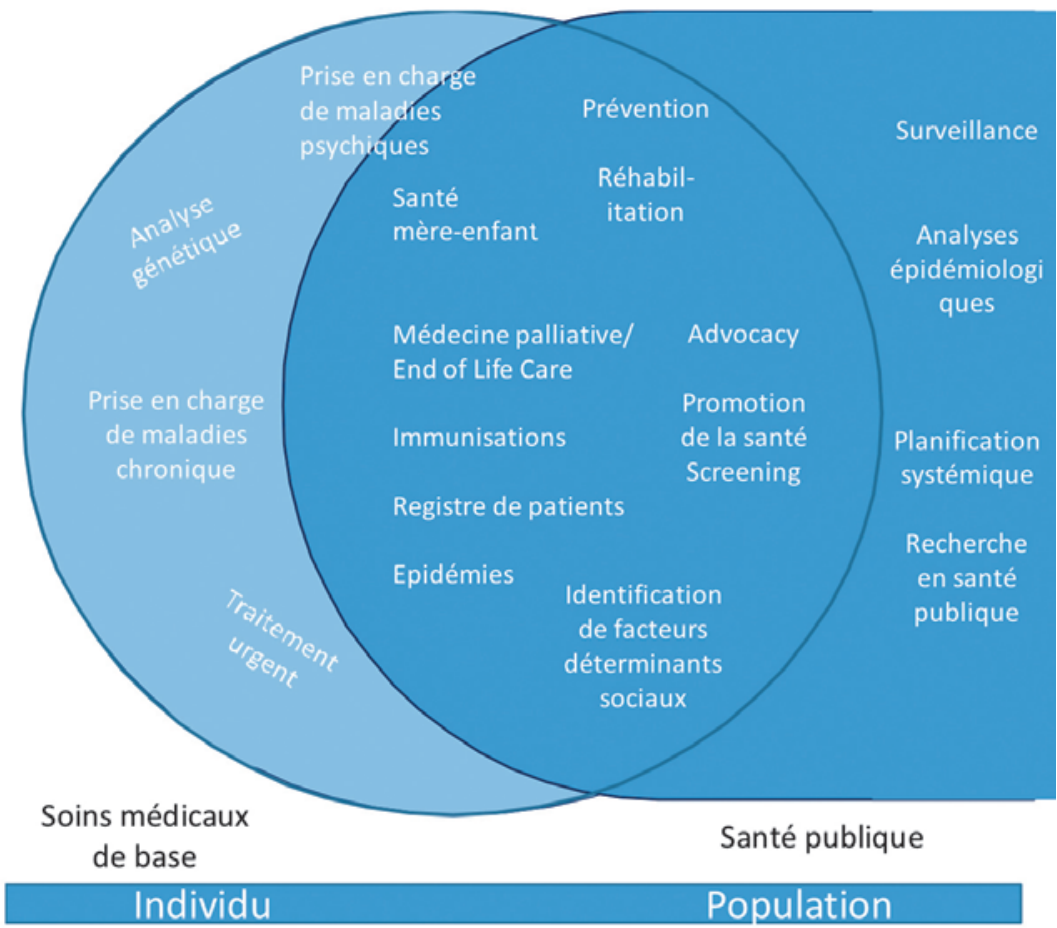

Care Continuum (AAFP)

Figure 1: Intersection des champs d'activité des soins médicaux de base et de la santé publique, adapté selon AAFP (AAFP 2017).
Health (MPH) ou d'un Master in Epidemiology (MSc Epidemiology). Cette formation continue théorique spécifique à la branche s'étend sur une année et approfondit les connaissances des candidat(e)s dans trois domaines thématiques: l'épidémiologie et la biostatistique (analyse de données et transfert dans la pratique), la prévention et la promotion de la santé (de l'analyse de problèmes à l'évaluation d'interventions), et le système de santé (politique de la santé, économie de la santé, droit de la santé).

Le nouveau programme de formation continue de notre société (révisé en 2017) se réfère aux compétences en santé publique recommandées par l'Association of Schools of Public Health in the European Region (ASPHER, https://www.aspher.org/). Ces contenus de formation reconnus au niveau international représentent aussi une référence utile pour la prise en compte de la diversité des parcours de formation.

\section{L'hétérogénéité croissante des formations}

théoriques constitue un défi que nous relevons volontiers.

L'hétérogénéité croissante des formations théoriques constitue un défi que nous relevons volontiers. Nous considérons cette diversité comme un enrichissement et souhaitons que le cercle de nos membres s'élargisse autour des médecins spécialistes en santé publique. Depuis peu, le Master en médecine des assurances est, par exemple, reconnu sous conditions en tant que formation continue théorique. Les médecins engagés dans la médecine des assurances peuvent, dès lors, se présenter à l'examen de médecin spécialiste en prévention et santé publique, pour autant qu'ils remplissent les autres critères de la formation continue.

Comme les soins médicaux de base, la santé publique occupe un rôle central dans le système de prise en charge médicale de la population. La définition qu'Acheson donne de la santé publique met en évidence les nombreux points communs qui relient les deux domaines. La santé publique est "the science and art of preventing disease, prolonging life and promoting health through the organized efforts of society" (Breda et al. 2015). Les médecins spécialistes en santé publique évoluent à différents niveaux du système de santé. D'une part, ils sont impliqués au niveau macro, dans la planification et le développement d'un système de santé tourné vers l'avenir, à l'échelle nationale, et, d'autre part, à un niveau méso, dans la mise en œuvre au niveau de la commune et du canton.

Au niveau micro, les spécialistes en santé publique vont à la rencontre d'individus et de groupes de population avec des besoins ou des risques particuliers, 
par exemple dans le cadre de projets de promotion de la santé ou dans le cadre d'enquêtes au niveau de la médecine scolaire. Les soins médicaux de base s'appliquent avant tout au niveau micro, où les spécialistes sont en contact direct avec les patientes et les patients et leur entourage: diagnostique, thérapie et prévention. Bien qu'un nombre important de domaines d'activité soient les mêmes pour la santé publique et les soins médicaux de base, les rôles et les fonctions dans ces domaines divergent (fig. 1). Les soins médicaux de base tendent à contribuer de plus en plus à des tâches générales comme le monitoring de la santé ou la promotion de la santé (voir stratégie NMT). Ainsi, de nouveaux champs d'activités communs s'ajoutent aux précédents.

Dès lors, la mise en réseau et la communication sont essentielles. C'est pourquoi nous entretenons des contacts étroits avec, notamment, le département de santé publique de la FMH, l'association des médecins cantonaux, l'association des médecins scolaires de Suisse, pour ne citer que quelques-unes des associations partenaires avec qui nous sommes en communication moyennant des formations communes et des échanges. Le contact régulier entre médecins de diverses disciplines devrait encore se renforcer à l'avenir, autant sur le plan du contenu que des politiques. C'est aussi pour cette raison que nous proposons aux médecins titulaires d'une autre spécialité et qui se considèrent comme des médecins actifs dans le domaine de la santé publique ou qui souhaitent soutenir ce domaine d'adhérer en tant que membres extraordinaires (http://sgpg.ch/sgpg_intro/devenir-membre/). Rassembler les différentes approches de la santé et de la maladie permet de tirer plus efficacement à la même corde lorsqu'il s'agit d'assurer un système de santé équitable et de qualité et de se protéger contre les risques de maladie.

La santé publique ne s'efforce pas seulement d'être interdisciplinaire, elle constitue aussi très clairement un domaine interprofessionnel. Et dans ce domaine, elle n'a pas attendu que l'interprofessionnalité devienne une mode ces dernières années. En Suisse, Santé publique Suisse (https://public-health.ch/fr/) est une organisation nationale et notre partenaire principal après la FMH. La collaboration avec la Swiss School of Public Health (https://ssphplus.ch/) revêt également une grande importance. Il s'agit d'une faculté interuniversitaire regroupant les huit universités suisses en vue de promouvoir les formations postgraduées et la recherche en santé publique. A l'étranger, nous sommes en contact avec la société autrichienne de santé pu-

La santé publique ne s'efforce pas seulement d'être interdisciplinaire, elle constitue aussi très clairement un domaine interprofessionnel.

blique, la société du Vorarlberg pour la santé publique, la société allemande de médecine sociale et de prévention ainsi que la société allemande de santé publique. La conférence bisannuelle "Public Helath ${ }^{3}$ " $\left(\mathrm{PH}^{3}\right.$, http://sgpg.ch/?s=PH3) offre la possibilité d'aborder les questions urgentes de santé publique sans considération de frontières. La prochaine édition de la $\mathrm{PH}^{3}$ aura d'ailleurs lieu à nouveau dans la région du lac de Constance en 2020. Toutes les personnes intéressées par la santé publique y sont cordialement invitées!

Si l'on veut être une voix qui porte dans la société, il faut de temps en temps savoir faire du bruit. Le meilleur moyen d'y arriver, pour une petite organisation spécialisée, est le chœur, c'est-à-dire la coopération. Ensemble avec toutes les organisations spécialisées, nous nous engageons pour un système de santé capable de relever les défis de demain et pour la santé de tous les groupes de population. Ce n'est que de cette façon que nous pourrons résoudre les enjeux de type médical, social, environnemental ou encore d'économie de la santé. La mise en commun des compétences de la médecine clinique et de la médecine des populations est la meilleure garantie, à court comme à long terme, d'une efficacité maximale.

\section{Références}

- American Academy of Family Physicians (AAFP). Integration of Primary Care and Public Health (Position Paper). 2017.

- Breda J, Wijnhoven T, Gabrijelčič M, Sigfrid L. Facets in Public Health in Europe. 2015.

- Berwick DM, Nolan TW, Whittington J. The triple aim: care, health, and cost. Health Aff (Millwood). 2008;27:759-69. 